\section{The ASYMMETRIC LEAVES complex maintains repression of KNOX homeobox genes via direct recruitment of Polycomb-repressive complex2}

\author{
Mukesh Lodha, Cristina F. Marco \\ and Marja C.P. Timmermans ${ }^{1}$
}

Cold Spring Harbor Laboratory, Cold Spring Harbor, New York 11724, USA

Polycomb-repressive complexes (PRCs) ensure the correct spatiotemporal expression of numerous key developmental regulators. Despite their pivotal role, how PRCs are recruited to specific targets remains largely unsolved, particularly in plants. Here we show that the Arabidopsis ASYMMETRIC LEAVES complex physically interacts with PRC2 and recruits this complex to the homeobox genes BREVIPEDICELLUS and KNAT2 to stably silence these stem cell regulators in differentiating leaves. The recruitment mechanism resembles the Polycomb response element-based recruitment of PRC2 originally defined in flies and provides the first such example in plants. Combined with recent studies in mammals, our findings reveal a conserved paradigm to epigenetically regulate homeobox gene expression during development.

Supplemental material is available for this article.

Received November 30, 2012; revised version accepted February 8, 2013.

Polycomb group (PcG) proteins are essential regulators of development in both plants and animals. PcG proteins form two functionally conserved complexes that maintain genes transcriptionally repressed by modulating chromatin structure. The Polycomb-repressive complex2 (PRC2) catalyzes the trimethylation of histone H3 Lys27 (H3K27me3), and PRC1, which binds this chromatin mark, mediates the monoubiquitylation of histone $\mathrm{H} 2 \mathrm{~A}$ and generates a somatically heritable compacted chromatin state (Schuettengruber and Cavalli 2009; Köhler and Hennig 2010; Margueron and Reinberg 2011; Bemer and Grossniklaus 2012). Genome-wide studies predict that as many as $20 \%$ of Arabidopsis genes are marked with H3K27me3 and regulated by PRC2 (Zhang et al. 2007a; Bouyer et al. 2011; Roudier et al. 2011). The precise spectrum of PRC2 targets varies depending on cell type and developmental stage and in response to external stimuli. How PRC2, which does not bind DNA specifically, recognizes defined targets is a major outstanding question

[Keywords: Polycomb proteins; homeobox genes; ASYMMETRIC LEAVES; stem cell; meristem; leaves]

${ }^{1}$ Corresponding author

E-mail timmerma@cshl.edu

Article published online ahead of print. Article and publication date are online at http://www.genesdev.org/cgi/doi/10.1101/gad.211425.112
(Köhler and Hennig 2010; Bemer and Grossniklaus 2012). In Drosophila, PRC2 is recruited to specific targets by DNA-binding proteins that bind conserved sequence motifs known as Polycomb response elements (PREs) (Schwartz et al. 2006; Schuettengruber and Cavalli 2009). In addition to PREs, long noncoding RNAs have been identified as important participants in Polycomb recruitment in mammals (Margueron and Reinberg 2011). Given the recent evidence that the noncoding RNA COLDAIR recruits PRC2 to FLOWERING LOCUS C (FLC) during vernalization (Heo and Sung 2011), PRC recruitment via long noncoding RNAs appears conserved between plants and animals. Whether the PRE-based recruitment mechanism is likewise conserved is unclear, as the mechanisms underlying the correct spatiotemporal recruitment of PRC2 to plant targets other than FLC are unknown.

Members of the class I KNOTTED1-like homeobox (KNOX) gene family are among the many predicted Polycomb targets identified in genome-wide H3K27me3 profiling studies (Zhang et al. 2007a; Bouyer et al. 2011; Roudier et al. 2011). KNOX genes promote stem cell activity, and their precise spatiotemporal regulation is essential to maintain the delicate balance between stem cell selfrenewal and differentiation (for review, see Barton 2010; Hay and Tsiantis 2010). KNOX activity is required in indeterminate cells within shoot meristems, the stem cell niches positioned at the growing tips of the plant, but needs to be stably repressed in determinate lateral organs, such as leaves, to allow cellular differentiation. The Arabidopsis KNOX family member SHOOTMERISTEMLESS (STM) is a verified Polycomb target (Schubert et al. 2006; Zhang et al. 2007b; Xu and Shen 2008; Bratzel et al. 2010), although the mechanism via which PRC2 is recruited to STM is not currently known. A role for PRC2 and PRC1 in the stable repression of the KNOX genes BREVIPEDICELLUS $(B P)$ and KNAT2 in leaves is less clear. H3K27me3 levels are enriched at these loci in whole seedlings and roots specifically. However, no extensive enrichment of LIKEHETEROCHROMATIN PROTEIN1 (LHP1), the chromodomain component of Arabidopsis PRC1, was observed at either locus in leaves (Zhang et al. 2007a,b; Roudier et al. 2011).

The persistent silencing of KNOX activity in determinate lateral organs may indeed involve distinct cellular memory systems. In contrast to the stable repression of $S T M$, silencing of BP and KNAT2 in developing leaves is mediated by ASYMMETRIC LEAVES1 (AS1) and AS2 (Lodha et al. 2008). These DNA-binding proteins bind as a complex to the promoters of $B P$ and KNAT2 and act after organ initiation to maintain both KNOX genes repressed throughout subsequent leaf development (Phelps-Durr et al. 2005; Guo et al. 2008). Here, we show that AS1 and AS2 physically interact with multiple core components of PRC2. The AS1-AS2 complex recruits PRC2 to $B P$ and KNAT2 to establish the somatically heritable repressed chromatin state required for leaf differentiation. The recruitment mechanism is reminiscent of the PREbased recruitment of PRC2 originally defined in flies (Schwartz et al. 2006; Schuettengruber and Cavalli 2009) and provides the first such example in plants. Combined with studies describing PRE-based Polycomb recruitment mechanisms in the regulation of homeobox genes in flies 
and, more recently, in mammals (Sing et al. 2009; Woo et al. 2010), our findings show that species-specific DNAbinding proteins have evolved to interact with the highly conserved PRC2 core components to direct the epigenetic repression of homeobox genes during development.

\section{Results and Discussion}

Polycomb complexes maintain repression of BP and KNAT2 in differentiating leaves

To assess the contribution of PRCs to the stable repression of BP and KNAT2, we compared the levels of H3K27me3 deposited at these KNOX loci in wild-type leaves and leaves mutant for the PRC2 core component CURLY LEAF (CLF) (Goodrich et al. 1997). In wild type, an enrichment for $\mathrm{H} 3 \mathrm{~K} 27 \mathrm{me} 3$ is detected at all regions examined across the BP and KNAT2 loci, with a localized peak of this repressive chromatin mark observed just downstream from the translation start site (Fig. 1). In clf, the levels of $\mathrm{H} 3 \mathrm{~K} 27 \mathrm{me} 3$ across both loci are strongly reduced. The residual $\mathrm{H} 3 \mathrm{~K} 27 \mathrm{me} 3$ observed at $B P$ and KNAT2 in clf mutants likely reflects redundancy with the H3K27 methyltransferase SWINGER (SWN), as seen for STM and other PRC2 targets in Arabidopsis (Schubert et al. 2006). Chromatin immunoprecipitation (ChIP) assays on chromatin isolated from seedling leaves expressing a functional LHP1-GFP fusion protein (Sung et al. 2006) showed that LHP1 also localizes to the promoter and coding region of both KNOX loci in leaves (Fig. 2). In addition, perturbation of PRC1 or PRC2 function leads to ectopic expression of STM as well as BP and KNAT2 (Supplemental Fig. 2; Katz et al. 2004; Xu and Shen 2008; Bratzel et al. 2010). These observations indicate that, as for STM, stable repression of BP and KNAT2 in leaves requires PRC2 and PRC1.

\section{Repressed KNOX loci lack heterochromatic marks}

In animals, Polycomb-mediated silencing of pluripotency factors, such as the homeobox genes Oct4 and Nanog, is likewise required to allow cellular differentiation (Margueron and Reinberg 2011; Orkin and Hochedlinger
2011). This initial repressed state is subsequently reinforced by methylation of H3K9 and DNA (Viré et al. 2006; Mikkelsen et al. 2008; Bhutani et al. 2010). To determine whether KNOX gene silencing in Arabidopsis leaves is similarly reinforced by establishment of a local heterochromatic state, we analyzed the distribution of H3K9me2 and cytosine methylation across $B P$ and KNAT2. Digestion with McrBC, which cleaves methylated DNA, did not significantly reduce the levels of amplifiable DNA, whether from the promoter or gene body of BP and KNAT2 (Supplemental Fig. 3A). This result is consistent with data from genome-wide methyl-cytosine profiling (Cokus et al. 2008) and suggests that both KNOX loci are largely unmethylated in leaves. Likewise, no significant enrichment of H3K9me2 was detected at either locus (Supplemental Fig. 3B). These data argue against a prominent role for heterochromatin-based gene silencing mechanisms in the stable repression of KNOX genes during leaf development, a finding consistent with observations that differentiated leaf cells are relatively easily reprogrammed to form new shoots or somatic embryos.

\section{The ASYMMETRIC LEAVES complex acts upstream of PRC2 in KNOX gene silencing}

Given that the stable repression of BP and KNAT2 in leaves involves the activities of the AS1-AS2 complex as well as PRC2, we asked whether these complexes function in a hierarchical manner. Toward this end, we compared the H3K27me3 profiles at BP and KNAT2 in wild-type, as1, and as2 leaves. As BP and KNAT2 misexpression in these mutants is most prominent in young leaves and at the base of expanded leaves (Supplemental Fig. 4; Guo et al. 2008), we compared H3K27me3 levels specifically in these tissues. Moreover, to correct for potential differences in nucleosome density across $B P$ and KNAT2 in wild type versus either mutant, H3K27me3 levels were normalized relative to histone $\mathrm{H} 3$ occupancy. In agreement with the data from whole seedling leaves (Fig. 1), enrichment for $\mathrm{H} 3 \mathrm{~K} 27 \mathrm{me} 3$ was observed at all sites tested across both KNOX loci in wild type (Fig. 2A). The levels of H3K27me3 peak just downstream from the proximal AS1-AS2-binding site, which, at BP, coincides with the location of enhancer elements that drive expression in developing leaves (Guo et al. 2008). In as1 and as2, H3K27me3 levels are significantly reduced at nearly all regions tested (Fig. 2A; Supplemental Fig. 1). Loss of this PRC2 signature is correlated with reduced PRC1 occupancy, as the enrichment levels of LHP1-GFP at BP and KNAT2 are significantly lower in as2 compared with wild type (Fig. 2B). Depletion of H3K27me3 in as1 is further correlated with increases in the levels of H3K4me3 (Fig. 2C), a chromatin mark deposited by Trithorax group proteins that act antagonistically to PcG complexes and promote gene expression (Carles and Fletcher 2009; Berr et al. 2010; Guo et al. 2010).

These experiments indicate that AS1 and AS2 act upstream of the Polycomb complexes in forming a repressive chromatin state at $B P$ and KNAT2 during leaf development. The observation that $B P$ and KNAT2 transcript levels in as1, clf, and the as1 clf double mutant are comparable (Supplemental Fig. 2D) is
Figure 1. CLF mediates the trimethylation of H3K27 at KNOX loci. ChIP experiments show that H3K27me3 levels across BP (left panel) and KNAT2 (right panel) are reduced in $c$ lf seedlings compared with wild type. Quantitative PCR values (mean $\mathrm{SE}$ ) are shown as percentage of input and calculated from at least three independent biological replicates. Schematic representations of the BP and KNAT2 loci are shown at the top. (Black dashes) Positions of amplicons analyzed in ChIP assays; (red ovals) AS1-AS2 complex-binding sites; (arrow) transcription start site; (light blue boxes) 5' and $3^{\prime}$ untranslated regions (UTRs); (dark blue boxes) ORF. Values on the $X$-axis are distances in kilobases relative to the transcription start site. 

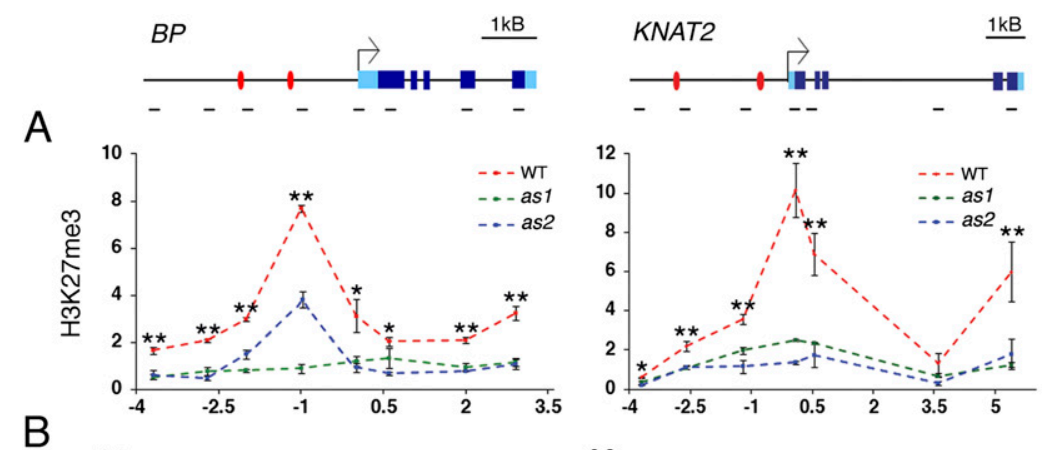

MYC-tagged version of CLF ( $p 35 S: M Y C-C L F$ ) in either a wild-type or as1 background. In nonmutant plants, CLF occupancy at $B P$ and KNAT2 is detected at regions surrounding the proximal AS1-AS2 complex-binding site (Fig. 3A), which include those BP and KNAT2 fragments marked by peak levels of H3K27me3 (Fig. 2A). Enrichment of CLF at these sites is comparable with that observed at AGAMOUS $(A G)$, which is a known direct target of CLFcontaining PRC2 (Schubert et al. 2006; Heo and Sung 2011). Failure to detect CLF binding above background at other sites across $B P$ and
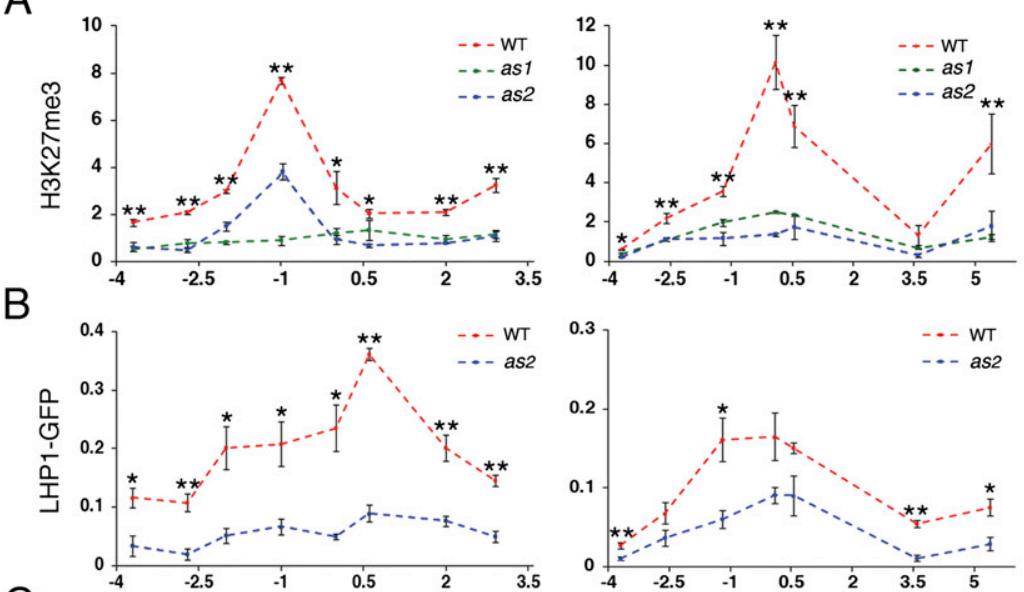

C
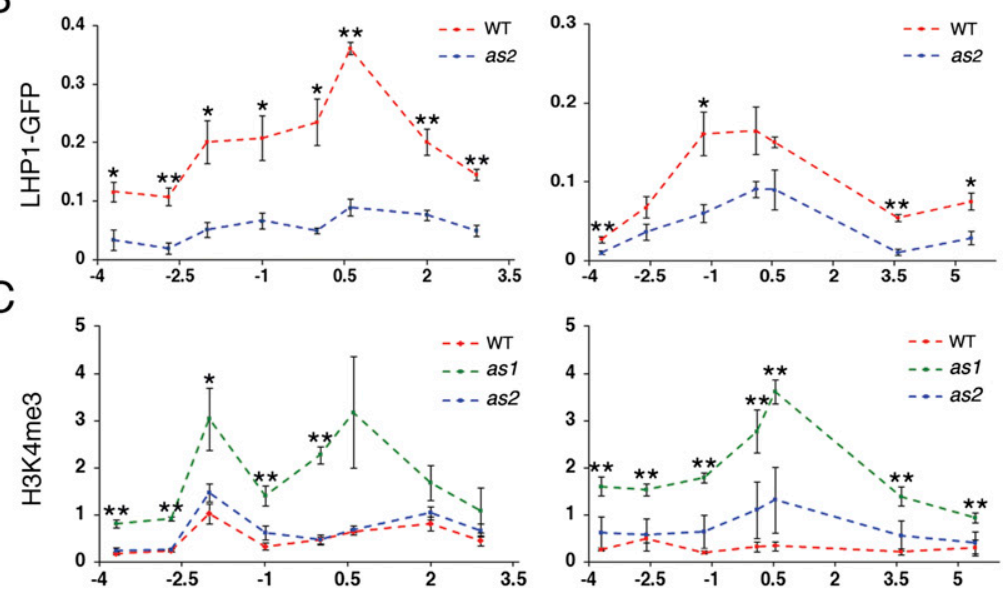

KNAT2, despite being marked by H3K27me3

(Fig. 2A), could suggest a relatively transient, dispersed association of PRC2 away from the proximal AS1-AS2 complex-binding site. Importantly, CLF occupancy at $B P$ and KNAT2 is dramatically reduced in as 1 (Fig. 3A), consistent with an AS1-AS2 complex-dependent recruitment of PRC2 to these target loci.

The possibility that the AS1-AS2 complex recruits PRC2 was substantiated by immunoprecipitation assays, which reveal an in vivo interaction between these complexes. In plants carrying an inducible YFP-tagged copy of AS2 (pOlexA:AS2-YFP), MYC-CLF is present in anti-GFP precipitates specifically following induction of AS2-YFP expression (Fig. 3B). Likewise, AS2-YFP is pulled-down in immunoprecipitation assays with anti-

Figure 2. The AS1-AS2 complex influences chromatin structure at BP and KNAT2. (A) Levels of H3K27me3 at BP (left panel) and KNAT2 (right panel) are significantly reduced in as1 and as2 leaves compared with wild type. (B) Occupancy levels of the PRC1 component LHP1 at BP (left panel) and KNAT2 (right panel) are likewise reduced in as2. (C) Enrichment levels for the active chromatin mark H3K4me3 across $B P$ (left panel) and KNAT2 (right panel) in wild type, as1, and as2. Values (mean $\pm \mathrm{SE}$; $n \geq 3)$ are normalized to $\mathrm{H} 3$ levels $(A, C)$ to correct for possible variation in nucleosome density at $B P$ and $K N A T 2$ or calculated as percentage of input $(B)$. Values significantly different from wild type in at least one of the mutants are indicated. $\left(^{\star}\right)$ $\left.P<0.05 ;{ }^{\star \star}\right) P<0.01$. Schematic representations of the BP and KNAT2 loci are as in Figure 1. Control experiments establishing the specificity and efficiency of ChIP reactions are shown in Supplemental Figure 1.

consistent with these complexes acting in the same pathway to repress both KNOX genes. However, the synergistic phenotypes of double mutants between as1, as2, and components of PRC2 or PRC1 indicate that these complexes also regulate distinct target sets (Supplemental Fig. 5). Considering this, it is unlikely that AS1 and AS2 effect the Polycomb-mediated repression of $B P$ and KNAT2 via regulation of PRC components. Indeed, the levels of $\mathrm{H} 3 \mathrm{~K} 27 \mathrm{me} 3$ in the overall histone $\mathrm{H} 3$ pool are unchanged in as1 and as2 (Supplemental Fig. 6). Moreover, H3K27me3 levels at STM, whose silencing in leaves occurs in an AS1-AS2 complex-independent manner, are unaffected in these mutants (Supplemental Fig. 6).

\section{The ASYMMETRIC LEAVES complex recruits PRC2 to KNOX loci by physical interactions}

Given the DNA-binding properties of AS1 and AS2 (Guo et al. 2008), their requirement for the Polycomb-mediated repression of $B P$ and KNAT2 could indicate that the AS1AS2 complex mediates the recruitment of PRC2 to these KNOX loci. This possibility was assessed using ChIP assays on chromatin prepared from plants expressing a
MYC but not using a nonspecific IgG antibody. An in vivo interaction between the AS1-AS2 and PRC2 complexes was also observed when $p O l e x A: A S 2-Y F P$ is coexpressed with an HA-tagged version of FERTILIZATION INDEPENDENT ENDOSPERM (FIE), the Arabidopsis ortholog of the Drosophila PRC2 component Extra sex combs (Esc) (pFIE:FIE$H A)$ (Köhler and Hennig 2010; Bemer and Grossniklaus 2012). FIE-HA is present in antiGFP precipitates only following induction, and AS2-YFP is specifically pulled down from total protein extracts in immunoprecipitation assays with anti-HA antibody (Fig. 3B).

Together, these observations support a model in which the AS1-AS2 complex physically interacts with PRC2 and recruits this complex onto the KNOX promoters. Deposition of $\mathrm{H} 3 \mathrm{~K} 27 \mathrm{me} 3$ at regions away from the PRC2 recruitment sites might then reflect spreading of PRC2 across $B P$ and KNAT2, which in animals is known to occur via an autocatalytic process mediated by binding of the PRC2 component Esc to H3K27me3 and subsequent activation of methyltransferase activity on H3K27 (Margueron et al. 2009; Suganuma and Workman 2010). Given that the propagation of PRC2 is likely to proceed discretely in individual cells, CLF occupancy beyond its sites of recruitment may be undetectable by ChIP.

To gain insight into the molecular mechanism via which the AS1-AS2 complex recruits PRC2 to $B P$ and $K N A T 2$, we next determined whether either DNA-binding protein interacts directly with PRC2 core components. The Arabidopsis genome includes one or more homologs for each of the Drosophila core PRC2 subunits. However, in the Arabidopsis seedling, the H3K27me3 


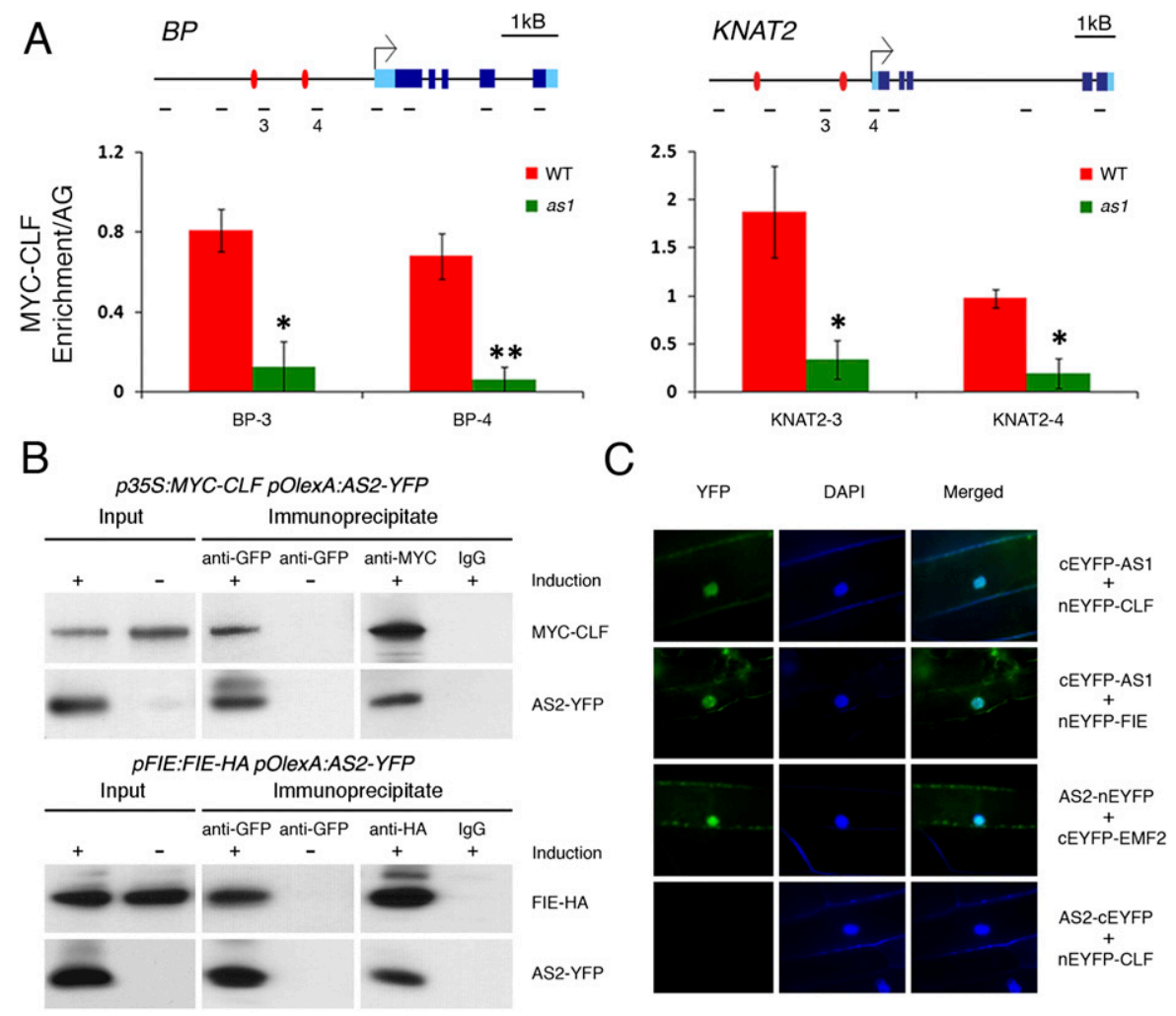

Figure 3. The AS1-AS2 complex physically interacts with PRC2 and directly recruits this complex to BP and KNAT2. (A) ChIP analyses showing CLF occupancy at specific regions of BP and KNAT2. CLF occupancy at these sites is significantly reduced in as 1 compared with wild type. $\left({ }^{*}\right) P<0.05 ;\left(^{\star \star}\right) P<0.01$. Values (mean $\pm \mathrm{SE} ; n \geq 3$ ) are relative to the enrichment of CLF at $A G$. $(B)$ In plants carrying an inducible AS2YFP fusion ( $p$ OlexA:AS2-YFP), MYC-CLF (top panel) and FIE-HA (bottom panel) coimmunoprecipitate with AS2-YFP specifically upon induction. AS2-YFP also coimmunoprecipitates with MYC-CLF (top panel) and FIE-HA (bottom panel) in immunoprecipitation assays with antiMYC and anti-HA antibody, respectively, but not in control immunoprecipitation assays with IgG. Antibodies used in immunoprecipitation assays are listed at the top, and proteins detected by Western are at the right. (C) Bimolecular fluorescence complementation assays reveal direct physical interactions between AS1 and the PRC2 core components FIE and CLF and between AS2 and EMF2. Cobombardment of functional AS2 and CLF fusion constructs (Supplemental Table 2) yields no fluorescence signal. (Left panels) EYFP signal monitoring protein-protein interactions. (Middle panels) DAPI staining indicating positions of nuclei. (Right panels) Merged images.

mark is predominantly deposited by a PRC2 complex that, in addition to the Enhancer of zeste $[\mathrm{E}(\mathrm{z})]$ homologs CLF or SWN, contains the Esc homolog FIE, the Suppressor of zeste $12[\mathrm{Su}(z) 12]$ homolog EMBRYONIC FLOWER2 (EMF2), and the P55 homolog MULTICOPY SUPPRESSOR IRA1 (MSI1) (Hennig and Derkacheva 2009). Bimolecular fluorescence complementation (BiFC) assays show that AS1 can physically interact with CLF and FIE, whereas AS2 interacts specifically with EMF2 (Fig. 3C; Supplemental Table 1).

\section{Recruitment of PRC2 to BP and KNAT2 involves a PRE-based mechanism}

Together, these data indicate that recruitment of PRC2 to $B P$ and KNAT2 is mediated by direct interactions with the AS1-AS2 complex, which in turn binds specific sequence motifs in the promoters of these homeobox genes (Guo et al. 2008). This recruitment mechanism is reminiscent of the PRE-based recruitment of PRC2 originally defined in flies (Schwartz et al. 2006; Schuettengruber and Cavalli 2009). However, a defining property of PREs is that these DNA elements are sufficient for the Polycomb-dependent repression of flanking genes at ectopic integration sites. To verify that intact AS1-AS2-binding sites are required for PRC2-mediated gene silencing and test whether a promoter element containing these sites has PRE-like activity, we generated multiple independent transgenic lines carrying a GFP-GUS reporter gene driven from a chimeric promoter containing a $B P$ promoter fragment with wild-type or mutated AS1-AS2binding sites upstream of the $35 \mathrm{~S}$ minimal promoter (Fig. 4). Leaf tissues from three independent transgenic lines containing either wild-type or mutated AS1-AS2binding sites in the promoter were pooled, and two pools for each were analyzed by ChIP. Using this strategy, H3K27me3 deposition at multiple independent loci could be evaluated simultaneously, and any effects of integration sites would be averaged. H3K27me3 levels are enriched at the GFP-GUS reporter in lines where the transgene contains intact AS1-AS2-binding sites in the promoter, and this enrichment is significantly reduced when the AS1-AS2-binding sites are mutated (Fig. 4; Supplemental Fig. 7). The AS1-AS2-binding sites are thus required and, in this context, also sufficient for the recruitment of PRC2 activity.

Considering these properties, we conclude that the AS1-AS2 complex recruits PRC2 to $B P$ and KNAT2 via a PRE-based mechanism, the first such example in plants. Given the role of COLDAIR in recruitment of PRC2 to 


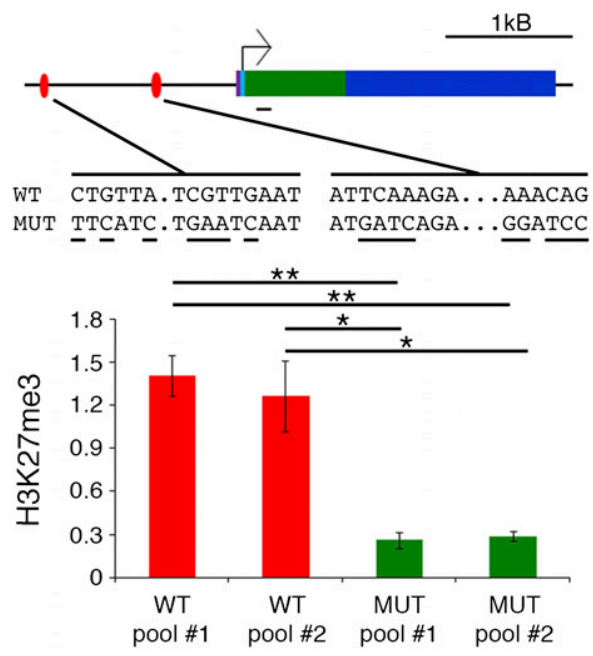

Figure 4. AS1-AS2-binding sites are necessary and sufficient for recruitment of PRC2 activity. ChIP analysis showing that H3K27me3 levels are enriched at the GFP-GUS reporter in lines where the transgene contains wild-type AS1-AS2-binding sites in the promoter. H3K27me3 levels are significantly reduced when the AS1-AS2binding sites are mutated. $\left(^{*}\right) P<0.05 ;\left(^{*}\right) P<0.01$. Two pools comprising three independent transgenic lines were analyzed for each construct. Quantitative PCR values (mean \pm SE) are normalized to $\mathrm{H} 3$ levels and calculated from three independent replicates. A schematic representation of the reporter lines is shown at the top. A BP promoter fragment spanning nucleotides -2707 to -1088 from the ATG was fused to the $35 \mathrm{~S}$ minimal promoter and inserted upstream of a GFP-GUS fusion. (Black dash) Position of the amplicon analyzed; (red ovals) AS1-AS2 complex-binding sites; (arrow) transcription start site; (violet box) the $35 \mathrm{~S}$ minimal promoter; (green box) GFP; (blue box) GUS. Sequence of the wild-type and mutated AS1AS2-binding sites are shown below. Controls establishing the specificity and efficiency of ChIP reactions are shown in Supplemental Figure 7.

FLC (Heo and Sung 2011), plants use at least two distinct Polycomb recruitment mechanisms: the one described here and a second involving long noncoding RNAs. As such, our findings show that, like the PRC2 core components themselves, the mechanisms via which this complex is recruited to specific targets are conceptually conserved between animals and plants.

How widespread PRE-based recruitment mechanisms are in plants remains to be seen. The genetic interactions between mutants affecting PRC2 and AS1-AS2 complex activity predict that this specific mechanism acts at a select subset of all Polycomb targets. Indeed, PRC2mediated silencing of STM and WUS occurs in an AS1AS2 complex-independent manner (Guo et al. 2008; Liu et al. 2011). Considering the broad repertoire of processes controlled by Polycomb proteins, diversity in PRC2 recruitment mechanisms is certainly expected. Likewise, animal Polycomb proteins are known to interact with multiple distinct recruitment proteins to generate target specificity (Schwartz et al. 2006; Schuettengruber and Cavalli 2009; Margueron and Reinberg 2011).

\section{A framework for Polycomb-mediated epigenetic repression of KNOX homeobox genes}

The AS1-AS2 complex binds two sites in the BP and KNAT2 promoters (Guo et al. 2008). These sites act nonredundantly, pointing to cooperativity between AS1AS2 complexes in the repression of these meristem regulators during organogenesis. A similar cooperativity has been reported for transcription factors acting at animal PREs (Schwartz et al. 2006; Schuettengruber and Cavalli 2009; Margueron and Reinberg 2011) and may be required to stabilize the binding of PRC2 at target loci. In this regard, it is also suggestive that AS1 and AS2 interact physically with multiple PRC2 core components. In addition, cooperativity between AS1-AS2 complexes could be important for the formation of long-range interactions between nucleosomes, which have been postulated to drive the switch from an active to a repressive chromatin state (Dodd et al. 2007; Angel et al. 2011). Consistent with this idea, binding of the AS1-AS2 complex at the KNOX promoters leads to a peak of $\mathrm{H} 3 \mathrm{~K} 27 \mathrm{me} 3$ deposition just downstream from the proximal AS1-AS2-binding sites. Once nucleated, positive feedback in PRC2 activity (Margueron et al. 2009; Suganuma and Workman 2010) is predicted to drive the propagation of this repressive chromatin mark across $B P$ and KNAT2. Autocatalytic activity of PRC2 along with PRC1 occupancy could further ensure that the repressive chromatin state is stably maintained through the many rounds of cell division associated with leaf development (Margueron et al. 2009; Bratzel et al. 2010; Suganuma and Workman 2010). Thus, even though AS1-AS2 complex activity is limited to early leaf development (Guo et al. 2008), repression of the KNOX genes persists throughout organogenesis. These findings thus provide a framework for the Polycomb-based cellular memory system underlying the somatically heritable repression of stem cell-promoting homeobox genes required for cellular differentiation in leaves. Combined with studies reporting PRE-based PRC2 recruitment mechanisms in the regulation of homeobox genes in flies (Schwartz et al. 2006; Schuettengruber and Cavalli 2009) and, more recently, also in mammals (Sing et al. 2009; Woo et al. 2010), this work reveals an ancient paradigm to control the spatiotemporal expression of homeobox genes during development.

\section{Materials and methods}

The ChIP experiments were performed as described previously (Guo et al. 2008). Relative enrichments were calculated as the percentage of input or as a ratio over $\mathrm{H} 3$ enrichment. In the MYC-CLF ChIP experiments, enrichments were calculated relative to the enrichment of CLF at AG. All experiments were performed at least three times independently, and PCR reactions were performed in duplicate. Student's $t$-test was used to calculate statistical significance. Protein immunoprecipitation reactions were performed using the $\mu$ MACS Epitope Tag isolation kits (Miltenyi Biotec). Tissues were processed without cross-linking, according to the manufacturer's recommendations, with one addition. Cleared sonicated lysates were treated with $5 \mu \mathrm{L}$ of benzonase $(250 \mathrm{U} / \mu \mathrm{L})$ to degrade DNA and RNA to avoid artificial protein-protein interactions. Samples were analyzed by Western as published previously (Guo et al. 2008). For BiFC assays, $2.5 \mu \mathrm{g}$ of each plasmid DNA was coated onto 1 $\mu \mathrm{M}$ gold particles and bombarded into onion epidermal cells using a PDS-1000/He Biolistic particle delivery system (Bio-Rad) as per the manufacturer's instructions. McrBC assays were performed at least three independent times. Approximately $500 \mathrm{ng}$ of genomic DNA was treated with $50 \mathrm{U}$ of $\mathrm{McrBC}$ (New England Biolabs) overnight at $37^{\circ} \mathrm{C}$. Control samples were treated identically but without enzyme. Amplifiable DNA levels were subsequently quantified by quantitative PCR using standard protocols. GUS staining was performed as described previously (Guo et al. 2008), and primers used in this study are listed in Supplemental Table 2. Further details can be found in the Supplemental Material. 


\section{Acknowledgments}

We thank Carrie Clendaniel and Carol Hu for technical assistance, Aman Husbands for the $p O l e x A: A S 2-Y F P$ line, Tim Mulligan for plant care, and the members of the Timmermans laboratory for helpful discussions and critical reading of the manuscript. We also thank R. Amasino and C. Helliwell for providing the p35S:LHP1-GFP and pFIE:FIE-HA seed, respectively. This work was supported by grants from the New York State Department of Health (C024308) and the National Science Foundation (MCB-0616114) to M.T.

\section{References}

Angel A, Song J, Dean C, Howard M. 2011. A Polycomb-based switch underlying quantitative epigenetic memory. Nature 476: 105-108.

Barton MK. 2010. Twenty years on: The inner workings of the shoot apical meristem, a developmental dynamo. Dev Biol 341: 95-113.

Bemer M, Grossniklaus U. 2012. Dynamic regulation of Polycomb group activity during plant development. Curr Opin Plant Biol 15: 118-125.

Berr A, McCallum EJ, Ménard R, Meyer D, Fuchs J, Dong A, Shen WH. 2010. Arabidopsis SET DOMAIN GROUP2 is required for H3K4 trimethylation and is crucial for both sporophyte and gametophyte development. Plant Cell 22: 3232-3248.

Bhutani N, Brady JJ, Damian M, Sacco A, Corbel SY, Blau HM. 2010. Reprogramming towards pluripotency requires AID-dependent DNA demethylation. Nature 463: 1042-1047.

Bouyer D, Roudier F, Heese M, Andersen ED, Gey D, Nowack MK, Goodrich J, Renou JP, Grini PE, Colot V, et al. 2011. Polycomb repressive complex 2 controls the embryo-to-seedling phase transition. PLoS Genet 7: e1002014.

Bratzel F, López-Torrejón G, Koch M, Del Pozo JC, Calonje M. 2010. Keeping cell identity in Arabidopsis requires PRC1 RING-finger homologs that catalyze H2A monoubiquitination. Curr Biol 20: 1853-1859.

Carles CC, Fletcher JC. 2009. The SAND domain protein ULTRAPETALA1 acts as a trithorax group factor to regulate cell fate in plants. Genes DeV 23: $2723-2728$.

Cokus SJ, Feng S, Zhang X, Chen Z, Merriman B, Haudenschild CD, Pradhan S, Nelson SF, Pellegrini M, Jacobsen SE. 2008. Shotgun bisulphite sequencing of the Arabidopsis genome reveals DNA methylation patterning. Nature 452: 215-219.

Dodd I, Micheelsen M, Sneppen K, Thon G. 2007. Theoretical analysis of epigenetic cell memory by nucleosome modification. Cell 129: 813822.

Goodrich J, Puangsomlee P, Martin M, Long D, Meyerowitz EM, Coupland G. 1997. A Polycomb-group gene regulates homeotic gene expression in Arabidopsis. Nature 386: 44-51.

Guo M, Thomas J, Collins G, Timmermans MCP. 2008. Direct repression of KNOX loci by the ASYMMETRIC LEAVES1 complex of Arabidopsis. Plant Cell 20: 48-58.

Guo L, Yu Y, Law JA, Zhang X. 2010. SET DOMAIN GROUP2 is the major histone $\mathrm{H} 3$ lysine 4 trimethyltransferase in Arabidopsis. Proc Natl Acad Sci 107: 18557-18562.

Hay A, Tsiantis M. 2010. KNOX genes: Versatile regulators of plant development and diversity. Development 137: 3153-3165.

Hennig L, Derkacheva M. 2009. Diversity of Polycomb group complexes in plants: Same rules, different players? Trends Genet 25: 414-423.

Heo JB, Sung S. 2011. Vernalization-mediated epigenetic silencing by a long intronic noncoding RNA. Science 331: 76-99.

Katz A, Oliva M, Mosquna A, Hakim O, Ohad N. 2004. FIE and CURLY LEAF polycomb proteins interact in the regulation of homeobox gene expression during sporophyte development. Plant $/$ 37: 707-719.

Köhler C, Hennig L. 2010. Regulation of cell identity by plant Polycomb and trithorax group proteins. Curr Opin Genet Dev 20: 541-547.

Lodha M, Marco CF, Timmermans MC. 2008. Genetic and epigenetic regulation of stem cell homeostasis in plants. Cold Spring Harb Symp Quant Biol 73: 243-251.

Liu X, Kim YJ, Müller R, Yumul RE, Liu C, Pan Y, Cao X, Goodrich J, Chen X. 2011. AGAMOUS terminates floral stem cell maintenance in Arabidopsis by directly repressing WUSCHEL through recruitment of Polycomb group proteins. Plant Cell 23: 3654-3670.

Margueron R, Reinberg D. 2011. The Polycomb complex PRC2 and its mark in life. Nature 469: 343-349.
Margueron R, Justin N, Ohno K, Sharpe ML, Son J, Drury WJ III, Voigt P, Martin SR, Taylor WR, De Marco V, et al. 2009. Role of the polycomb protein EED in the propagation of repressive histone marks. Nature 461: 762-767.

Mikkelsen TS, Hanna J, Zhang X, Ku M, Wernig M, Schorderet $\mathrm{P}$, Bernstein BE, Jaenisch R, Lander ES, Meissner A. 2008. Dissecting direct reprogramming through integrative genomic analysis. Nature 454: 49-55.

Orkin SH, Hochedlinger K. 2011. Chromatin connections to pluripotency and cellular reprogramming. Cell 145: 835-850.

Phelps-Durr TL, Thomas J, Vahab P, Timmermans MCP. 2005. Maize rough sheath2 and its Arabidopsis orthologue ASYMMETRIC LEAVES1 interact with HIRA, a predicted histone chaperone, to maintain knox gene silencing and determinacy during organogenesis. Plant Cell 17: 2886-2898.

Roudier F, Ahmed I, Bérard C, Sarazin A, Mary-Huard T, Cortijo S, Bouyer D, Caillieux E, Duvernois-Berthet E, Al-Shikhley L, et al. 2011. Integrative epigenomic mapping defines four main chromatin states in Arabidopsis. EMBO J 30: 1928-1938.

Schubert D, Primavesi L, Bishopp A, Roberts G, Doonan J, Jenuwein T, Goodrich J. 2006. Silencing by plant Polycomb-group genes requires dispersed trimethylation of histone $\mathrm{H} 3$ at lysine 27. EMBO I 25: 4638-4649.

Schuettengruber B, Cavalli G. 2009. Recruitment of polycomb group complexes and their role in the dynamic regulation of cell fate choice. Development 136: 3531-3542.

Schwartz YB, Kahn TG, Nix DA, Li XY, Bourgon R, Biggin M, Pirrotta V. 2006. Genome-wide analysis of Polycomb targets in Drosophila melanogaster. Nat Genet 38: 700-705.

Sing A, Pannell D, Karaiskakis A, Sturgeon K, Diabali M, Ellis J, Lipshitz HD, Cordes SP. 2009. A vertebrate Polycomb response element governs segmentation of the posterior hindbrain. Cell 138: 885-897.

Suganuma T, Workman JL. 2010. WD40 repeats arrange histone tails for spreading of silencing. J Mol Cell Biol 2: 81-83.

Sung S, He Y, Eshoo TW, Tamada Y, Johnson L, Nakahigashi K, Goto K, Jacobsen SE, Amasino RM. 2006. Epigenetic maintenance of the vernalized state in Arabidopsis thaliana requires LIKE HETEROCHROMATIN PROTEIN 1. Nat Genet 38: 706-710.

Viré E, Brenner C, Deplus R, Blanchon L, Fraga M, Didelot C, Morey L, Van Eynde A, Bernard D, Vanderwinden JM, et al. 2006. The Polycomb group protein EZH2 directly controls DNA methylation. Nature 439: 871-874.

Woo CJ, Kharchenko PV, Daheron L, Park PJ, Kingston RE. 2010. A region of the human HOXD cluster that confers polycomb-group responsiveness. Cell 140: 99-110.

Xu L, Shen WH. 2008. Polycomb silencing of KNOX genes confines shoot stem cell niches in Arabidopsis. Curr Biol 18: 1966-1971.

Zhang X, Clarenz O, Cokus S, Bernatavichute YV, Pellegrini M, Goodrich J, Jacobsen SE. 2007a. Whole-genome analysis of histone H3 Lysine 27 trimethylation in Arabidopsis. PLoS Biol 5: e129.

Zhang X, Germann S, Blus BJ, Khorasanizadeh S, Gaudin V, Jacobsen SE. 2007b. The Arabidopsis LHP1 protein colocalizes with histone H3 Lys27 trimethylation. Nat Struct Mol Biol 14: 869-871. 


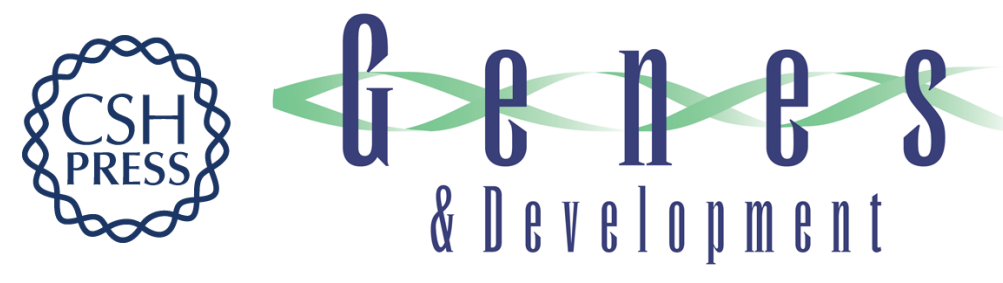

\section{The ASYMMETRIC LEAVES complex maintains repression of KNOX homeobox genes via direct recruitment of Polycomb-repressive complex2}

Mukesh Lodha, Cristina F. Marco and Marja C.P. Timmermans

Genes Dev. 2013, 27: originally published online March 6, 2013

Access the most recent version at doi:10.1101/gad.211425.112

Supplemental http://genesdev.cshlp.org/content/suppl/2013/02/27/gad.211425.112.DC1

Material

References This article cites 37 articles, 10 of which can be accessed free at:

http://genesdev.cshlp.org/content/27/6/596.full.html\#ref-list-1

License

Email Alerting Receive free email alerts when new articles cite this article - sign up in the box at the top

Service right corner of the article or click here.

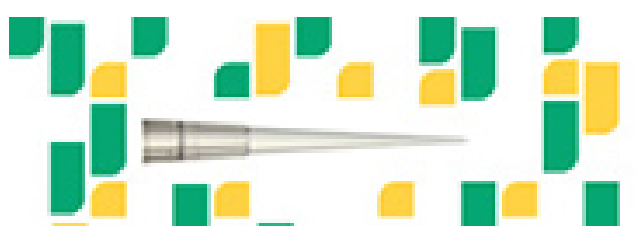

Focused on your science. 